\title{
DAMAGE DETECTION OF CONCRETE GRAVITY DAMS USING HILBERT-HUANG METHOD
}

\author{
Sajad Esmaielzadeh $^{\text {a }}$, Hassan Ahmadi ${ }^{b} *$, Seyed Abbas Hosseini ${ }^{\mathrm{c}}$ \\ ${ }^{a}$ Department of Civil Engineering, Science and Research Branch, Islamic Azad University, Tehran, Iran, \\ E-mails: sajad_59es@yahoo.com, abbas_hoseyni@srbiau.ac.ir \\ b* Department of Civil Engineering, Roodehen Branch, Islamic Azad University, Tehran, Iran, e-mail: hassanahmadi551@gmail.com
}

Received: 26.05.2018 / Accepted: 05.09.2018 / Revised: 23.10.2018/ Available online: 15.12.2018

DOI: 10.2478/jaes-2018-0012

KEY WORDS: structural damage, damage index, modal shapes, signal processing, relative frequency error.

\begin{abstract}
:
Damage detection in concrete gravity dams using Hilbert-Huang Method, as one of the most common signal processing techniques, is studied in this research. After considering a typical geometry for dams, damage is modelled by a reduction in the modulus of elasticity in the dam's body (in three levels of damage) and in different areas of the structure. The dam is excited by a horizontal earthquake and the primary natural frequencies of the dam are calculated by applying Hilbert-Huang Method to the measured response, which is the acceleration of five points in the system. Based on the changes in the frequencies of the damaged and undamaged structure, a parameter, called relative frequency error, has been introduced. The results show that the proposed criterion used in this study can not only properly identify the location of damage but also predict the severity of the structural damage in concrete gravity dams accurately.
\end{abstract}

\section{INTRODUCTION}

Concrete gravity dams are valuable sources for each country and any failure in them can cause economic problems and loss of life. The probability of failure during the operation of a structure has a variety of reasons, including lack of exact compliance of regulations at the time of structural design, disregarding the correct principles in construction, lack of proper maintenance, passage of time and abnormal loadings, such as earthquakes and wind. If partial damages to a structure cannot be detected in time, they can cause a general failure in the structure. In this situation, according to the degree of importance of the structure, it is required that the essential information on the situation of structural health and damage severity be acquired in order to safeguard and strengthen the structure as soon as possible (Kyang, 2005).

In the area of structural health monitoring, failure is defined as changes in the physical parameters of the model. Due to the relationship between physical parameters and dynamic properties of the model, it seems that damages can be detected from changes in the dynamic parameters which are fundamental concepts for vibration-based methods. In addition, damage detection is defined as all methods and techniques to detect the presence and position of damage, which can indicate the failure rates (Doebling et al., 1998). In this context, Rytter has classified the methods of damage detection into four categories, including determining the existence of damage in the structure, specifying the coordinates of the location of damage, highlighting the severity of damage to the structural members and estimating the remaining life of serviceability (Rytter, 1993). For detection of damage in structures, if visible, one can use direct observation, but there are often limitations which force the use of tests on these structures. These tests are divided into two categories: destructive and non-destructive. Obviously, the second category is more acceptable due to a lack of damage (Chang et al., 2003). For example, by inserting some sensors on a bridge (without causing damage to the structure) and recording the structural response using signal processing techniques (such as Wavelet Method) under ambient vibration, the presence or absence of damage can be realized (Richard, 2010).

Numerous researches using non-destructive techniques, like signal processing methods, have been studied so far, but the investigated structures mainly consist of simple structural elements, such as frames, beams, and trusses, which enjoy much easier governing equations compared to the dams. Apparently, the cause of less attention to this issue can be regarded as complexity in modelling and analysis and considering the interaction among different bodies, as well as the high degree of freedom of the dams in comparison with other structures (Chopra, 2014).

\footnotetext{
* Corresponding author: Hassan AHMADI, Assistant professor, e-mail: hassanahmadi551@gmail.com
} 
In this section, a brief review is conducted in the studies of damage detection in concrete gravity dams. Cleary et al. presented a non-mesh method for modelling of the damage in gravity dams under earthquake loading. The proposed method was called $\mathrm{SPH}^{1}$ and was implemented on the Koyna Dam under severe harmonic ground excitation. The results of this method involve the formation and distribution of cracks, which shows a good agreement between the Finite Element Method and experimental results. Moreover, they found that the amplitude and frequency of excitation extremely affect the pattern of failure (Cleary, 2013). Alembagheri and Ghaemian studied the damage assessment of a concrete arch dam (the Morrow Point Dam) through nonlinear IDA ${ }^{2}$, which includes the dam-foundation interaction. The conducted study introduces two damage indices based on Maximum Crest Displacement $\left(\mathrm{DI}_{\mathrm{U}}\right)$ and Damage Energy Dissipation $\left(\mathrm{DI}_{\mathrm{E}}\right)$. They showed that the proposed damage indices can properly assess the damage of the dam (Alembagheri et al., 2013). Bukenya et al. in a review article investigated the researches on the health monitoring of concrete dams, which includes the static and dynamic behavior of dams. Familiarizing the enthusiasts with the techniques used in this field is the main objective of this paper. Furthermore, as a part of this article, the areas of possible future work on the health monitoring of concrete dams is discussed (Bukenya et al., 2014) Chen and Zhang also studied the initiation of cracks in the crest of concrete arch dams' galleries using the Finite Element Method. They found that the weight of the dam has a greater impact on the stresses of the arch crests of the galleries in high concrete dams (Chen et al., 2014). Finally, it can be pointed to the work done by Aldemir et al. who investigated the dynamic response of the Melen Dam using a 1:75 scale model. They studied the crack propagation, sliding displacement and stability of dams and showed that there is a good agreement between experimental and analytical results (Aldemir et al., 2015).

With an overview of the investigations, which have been done on damage detection of dams, it appears that there is a lack of usage of signal processing method in this field. The main aim of this study is to use Hilbert-Huang Method, as one of the most common techniques in signal processing, in order to predict the location and severity of probable damages in concrete gravity dams without causing damage to the structure using the information from the sensors installed on dams. The response of the structure is assumed as the output acceleration in five points in the height of the dam. In addition, two damage indices are proposed based on the difference between the natural frequency of the healthy and damaged structures.

\section{DAMAGE DETECTION METHODS}

As it was previously mentioned, structural damage detection methods are generally divided into two categories: destructive and non-destructive. Obviously, in order to prevent structural damage, the usage of non-destructive methods is preferable. On the whole, non-destructive methods are divided into two classes of static and dynamic. Static methods are based on measurement of displacement, strain of structures under static loads and the use of updated Finite Element models. These methods are

\footnotetext{
${ }^{1}$ Smoothed Particle Hydrodynamics

${ }^{2}$ Incremental Dynamic Analysis
}

widely used for monitoring the damage assessment of bridges, even though they have some limitations, such as inefficiency at the time of static loading tests, lack of an immediate review of the structure and the high volumes of measured data (Alvandi et al., 2006). Dynamic methods, which enjoy more application than the static ones in this area, are based on variation of vibrational characteristics. These methods are based on the principle that damage causes changes in the dynamic properties of a structure. It should be noted that dynamic methods can be divided into two categories: methods based on modal parameters and those based on signals.

\subsection{Methods Based on Modal Parameters}

In these methods, changes in the measured modal parameters, such as frequencies, modal damping ratio and mode shapes (or their derivatives), are considered as signs of change in the physical (dynamic) properties such as mass, stiffness and damping. Changes in the natural frequencies are much of the earlier studies in order to detect structural damage in the field of dynamic methods. In a study by Salawu, a detailed review on this class of methods was implemented (Salawu, 1997).

Generally, these kinds of studies involve two general categories, including direct and inverse problems. The direct method can only detect the presence or absence of damage, but in the inverse method, in addition to detecting the possibility of failure, there is also the possibility of determining the location and severity of damage. Although the response of the structure contains information other than natural frequencies, structural damage detection using natural frequencies is easier than investigation of mode shapes and modal damping (Osegueda, 1992). Several studies have evaluated the effect of downtime on damage detection. Another category of research is based on the changes in mode shapes of the structure. For example, MAC ${ }^{3}$ and $\mathrm{COMAC}^{4}$ are included in this category to compare the similarity of mode shapes before and after damage. In this context, Fox shows that a common single-valued criterion (such as MAC), which is used to compare mode shapes, has a low sensitivity to damage (Fox, 1992). It should be noted that in order to create more sensitive measured parameters, instead of using mode shapes, their derivatives can be used to evaluate the changes in the vibrational behavior of the structures.

In this regard, Pandey, using the Finite Element model of a beam, shows that change in the curvature of mode shapes is an appropriate parameter for damage detection (Pandey et al., 1991). There are also various other methods, the concepts of which are presented as: change in modal strain energy of the structural elements (Stubbs et al., 1992 and Alvandi et al., 2006), measurement of dynamic flexibility (Patjawit et al., 2005), comparison of frequency response functions before and after damage (Heylen, 1996) and using Artificial Intelligence methods, such as Neural System, Fuzzy Logic, ANFIS and Genetic Algorithm (Doebling et al., 1998; Chang et al., 1999; Jeyasehar et al., 2006; Heylen et al., 1996; Zhu et al., 2014 and Ruotolo et al., 1997).

\footnotetext{
${ }^{3}$ Modal Assurance Criterion

${ }^{4}$ Co-ordinate Modal Assurance Criterion
} 


\subsection{Signal Processing Methods}

The purpose of signal processing methods is evaluation of the features which are gained directly from the time histories or corresponding spectra. In these methods, using signal processing tools, the hidden features in the signal are extracted. In most cases in civil structures, structural response is the same signal that is recorded by sensors in various locations. The responses are often caused by environmental changes, such as wind, earthquake and so on. In addition, the output signal or signals are the results of sensors which are located in different parts of the structure. In most cases, acceleration sensors are used to evaluate the structural responses (due to availability, sensitivity and cheapness) and signal processing is applied on these acceleration responses. Generally, these methods often occur in two main processes:

- $\quad$ Recording a large amount of data from different parts of structures

Selecting features from data that are associated with the damage

In these methods, large volumes of data are often witnessed. Thus, compared with other methods, the need to properly condensate and interpret the data is necessary. Overall, these methods are divided into three categories:

- Time domain methods

Frequency domain methods

Time-Frequency domain methods

Time domain is one of the most common methods of signal processing for time series analysis (a time series is a series of data points listed in the time order). In this method, vibration of structure, which could include acceleration, velocity or displacement, is investigated. Afterwards, by extracting the sensitive parameters to damage from the recorded response, structural health is monitored.

Frequency domain techniques are based on the frequency content which tries to identify the structural damage. The most appropriate tool for this method can be named as spectral Fourier transform analysis and frequency response technique. Fourier transform of a signal takes it from time-space to frequency-space and by using it, the amplitude of different frequencies can be observed. Often, the frequency in which the amplitude is more frequent and larger will determine the fundamental mode of structure (Rusch et al., 1995).

Time-frequency domain methods completely eliminate time. Therefore, these methods may not be applicable to nonstationary signals. Due to these shortcomings, some methods were invented to provide information in the frequency domain together with some data in the time domain. Among these methods, there are short-time Fourier transform, Wavelet transforms and Hilbert-Huang Method. Fourier transforms can be named as the source for all these methods. In other words, all techniques emerging after Fourier transform try to fix its shortcomings. In fact, Fourier transform, by eliminating time, exhibits the changes of amplitude in different frequencies. The following formula is a mathematical expression of Fourier transform:

$x(f)=\int_{-\infty}^{+\infty} x(t) e^{-i 2 \pi f t} d t$
In the above equation $e^{-i 2 \pi f t}$ is:

$e^{-i 2 \pi f t}=\cos (2 \pi i f t)-i \sin (2 \pi i f)$

As it was mentioned above, the disadvantage of this method is that it is never known at what time, what the frequency of vibration of the structure is. In other words, during the transformation, the time domain is lost and there will be only two variables, including frequency and amplitude. Of course, this problem has partially been resolved using methods, such as Wavelet transform and short-time Fourier transform (Brincker, 2001 and Lee et al., 2007). In order to avoid the shortcomings of Fourier transform, other methods are also provided, such as Hilbert-Huang transform method since Fourier transform is originally defined for stationary data analysis and usage of this method for non-stationary data is occasionally incorrect. Fourier spectrum, which defines the harmonic components, is constant over time; thus, in determining the frequency content of time, it is not reliable. In short-time Fourier transform, to eliminate the disadvantages, the signal is multiplied by a function with limited domain in both the time and frequency domains, which is called window function. In this way, there is a small piece of signal that can be assumed as a stationary one. Then the Fourier transform is applied. Similarly, by shifting the window function through the signal, there will be a transform with two output variables in terms of frequency and time. In order to detect high frequencies, a wider window function is needed and for lower frequencies, a thinner window function is required, but in the short-time Fourier transform, the window function is constant and this is a problem with this approach (Allen, 1977). Wavelet transform, similar to Fourier analysis, divides the signal into several curves with different details, but the difference is that, unlike Fourier analysis, it is not decomposed to Sine-waves; it becomes a coefficient of a base signal or mother wavelet function, which has a finite length and an average value equal to zero (Farsayi et al., 2010). Some of this mother wavelet functions are shown in Figure 1. For more information in this context, refer to the following references (Balafas et al., 2015; Min et al., 2011 and Carolina et al., 1999):

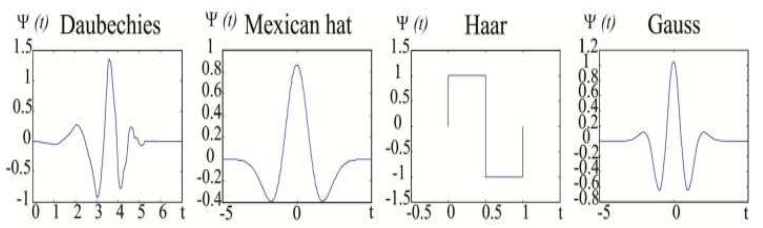

Figure 1. Some of the Mother Wavelets (Farsayi et al., 2010)

\subsection{Hilbert-Huang Transform}

Huang et al. developed a new method for signal processing which is one of the most widely used methods in this field because of the ability to analyze the non-stationary signals. This technique tries to use a combination of the Empirical Mode Decomposition Method and Hilbert transform in order to extract the amplitude and frequency of a signal over time. The combination of the two mentioned methods is known as the Hilbert-Huang Method (Chen et al., 2014 and Huang et al., 1998). In this method, the signal is decomposed to some Intrinsic Mode Functions (IMF) that can show time-frequency (using the Hilbert transform) and consequently obtain the modal characteristics (Tang et al., 2011; Amiri et al., 2015 and Ramezani et al., 2010). 
Hilbert transform is a mathematical linear operator which is applicable to signal processing. Through using this technique, the analytical signal of a given signal can be found. According to Cauchy's Integral, the Hilbert transform of a signal of $x(t)$ is defined as:

$$
H[x(t)]=y(t)=\frac{1}{\pi} P V \int_{-\infty}^{+\infty} \frac{x(t)}{t-\tau} d \tau
$$

In fact, this transformation shifts the value of the phase to the amount of $0.5 \pi$. Using this definition, the analytical form of a signal $x(t)$ is equal to $z(t)$, and its conjugate in complex space is $y(t)$, which is formulated as follows:

$$
\begin{aligned}
& z(t)=x(t)+i y=a(t) e^{i \theta(t)} \\
& a(t)=\sqrt{x^{2}(t)+y^{2}(t)} \\
& \theta(t)=\arctan \left[\frac{y}{x}\right]
\end{aligned}
$$

Finally, for calculating the instantaneous frequency, one can write:

$f(t)=\frac{1}{2 \pi} \frac{d \theta(t)}{d t}$

According to the above definitions, Hilbert transform provides a unique method to define the imaginary part of a signal. Moreover, it should be noted that Hilbert transform is faced with shortcomings. For example, one can refer to Bedrosian's theorem (Bedrosian, 1963). According to Bedrosian's theorem, Hilbert transform of a function with real part is written as follows:

$$
\operatorname{Re}(z(t))=x(t)=a(t) \cos (\theta(t))
$$

Only if the Fourier spectra have not shared the frequency range, which is the product of the two functions, the range of frequency $\cos (\theta(t))$ will be higher than frequency range $a(t)$. This can be written as:

$$
H[a(t) \cos (\theta(t))]=a(t) H[\cos (\theta(t))]
$$

If this condition cannot be met, phase and amplitude changes will be mixed together and determining the frequency accurately will be impossible. In other words, the rate of changes in the phase function should be greater than that in the amplitude function for frequency identification and the variation of phase will be found. Therefore, some methods were invented to provide signals for using Hilbert transform in order to reduce errors caused by this method. In this case, Empirical Mode Decomposition (EMD) method can be referred to (Huang et al., 1998). As it was previously mentioned, one of the common tools for preparing the original signal for Hilbert transform is the EMD technique, the name of which properly expresses its nature. In this method, through using an empirical method, a signal is decomposed to some signals, each of which has almost constant frequency content and its divided signals are called Intrinsic Mode Function (IMF). In other words, the original signal can be derived from the sum of all IMFs. It should be noted that, unlike harmonic waves, the amplitude and frequency of Intrinsic Mode Functions is changeable at the time. This behavior causes the Intrinsic Mode Functions to change into non-stationary signals which lead to a desired Hilbert transform. For this, there are two basic assumptions:

- For the given data, the number of maximum points should be equal to the number of zero points. This assumption is used in many other methods and its physical concept establishes the condition for oscillation of a signal.

- At each point of data, average of maxima and minima is equal to zero. Physical concept of this assumption is the symmetric condition of a signal. As a result, this assumption will prevent the asymmetric oscillations that are not desirable (Huang et al., 1998).

The following are the required steps for Empirical Mode Decomposition (Rilling et al., 2003):

- Finding the local extremums (local maximums and minimums)

- Passing a Spline through the maximum points

- Passing a Spline through the minimum points

- Determining the average of the two curves defined above ${ }_{\text {as }} m_{i, j}$, in which $i$ relates to the number of Intrinsic Mode Function and $j$ denotes the number of iteration for obtaining the $\mathrm{i}^{\text {th }}$ Intrinsic Mode

- Subtracting $m_{i, j}$ from original signal and obtaining the first component of $h_{j}$ :

$h_{i, j}=x-m_{i, j}$

Or for subsequent steps:

$h_{i, j}=r_{i}-m_{i, j}$

- Repeating the above process and naming the remaining signal as $h_{j+1}$ (each time $j$ increases by one):

$h_{i, k}=h_{i, j-1}-m_{i, j}$

- In each iteration for step 5, a stopping criterion is considered

- The last remaining signal is defined as the first IMF:

$C_{i}=h_{i, k}$

- Subtracting the first IMF from the original signal:

$r_{i+1}=x-C_{i}$

- The obtained signal from step 9 is substituted into step 1 and then the process is repeated (in all of the above steps, the first index represents the number of IMF). Eventually, the original signal can be decomposed as following:

$x=\sum_{i=1}^{n} C_{i}+r_{n+1}$ 


\section{NUMERICAL MODELLING}

In this section, a typical concrete gravity dam with the height of $30 \mathrm{~m}$ as shown in Figure 2 is considered:

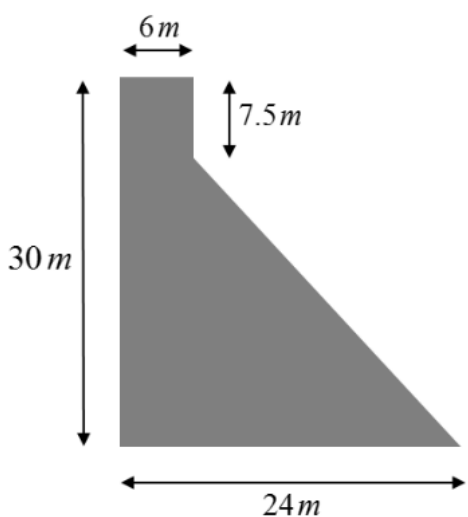

Figure 2. Geometry of the Considered Concrete Gravity Dam

In the modelling of the structure, the length of the reservoir is assumed to be $150 \mathrm{~m}$ ( 5 times the height of the dam) and the foundation is assumed to be rigid (Figure 3). In addition, for convenience in the modelling of the dam-reservoir interaction, the free board height is neglected and the interaction is considered by tying the nodes of the interface (tie interaction). The far-end boundary condition of the reservoir is also supposed as a type of planar and non-reflecting (Humar et al., 1988).

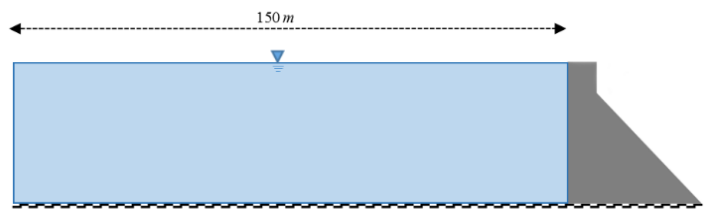

Figure 3. Dam and Reservoir in Modelling

Rayleigh-Damping is used in the definition of damping in which the coefficient of mass and stiffness are obtained from frequency analysis (Bathe, 1996). Also, the material properties are according to Table 1 .

Table 1. Some of the Material Properties Used in Analysis of

\begin{tabular}{|c|l|l|c|}
\multicolumn{4}{c}{ the Dam } \\
\hline Parameter & Symbol & Value & Unit \\
\hline $\begin{array}{c}\text { Modulus of } \\
\text { Elasticity of Dam }\end{array}$ & $E_{c}$ & 25 & $G P a$ \\
\hline $\begin{array}{c}\text { Specific Weight } \\
\text { of Dam }\end{array}$ & $\gamma_{c}$ & 24 & $\mathrm{kN} / \mathrm{m}^{3}$ \\
\hline $\begin{array}{c}\text { Specific Weight } \\
\text { of Reservoir }\end{array}$ & $\gamma_{w}$ & 9.81 & $\mathrm{kN} / \mathrm{m}^{3}$ \\
\hline $\begin{array}{c}\text { Bulk Modulus of } \\
\text { Reservoir }\end{array}$ & $K_{w}$ & 2.2 & $G P a$ \\
\hline
\end{tabular}

According to Figure 4, the horizontal component of El-Centro Earthquake, which is scaled to $0.3 \mathrm{~g}$, has been used for structure loading.

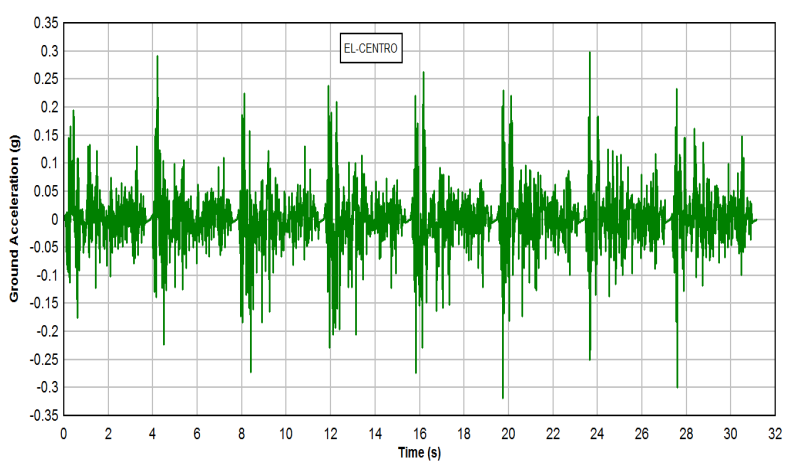

Figure 4. Horizontal Component of El-Centro Earthquake

In this research, damage is considered as the reduction of modulus of elasticity (or the softening of the damaged areas). Therefore, three levels of damage are defined according to Table 2 .

Table 2. Damage Indexes

\begin{tabular}{|c|c|c|}
\hline Damage Index & $\begin{array}{c}\text { Reduction of } \\
\text { Stiffness }\end{array}$ & $\begin{array}{c}\text { Corresponding } \\
\text { Modulus of } \\
\text { Elasticity }\end{array}$ \\
\hline $\mathrm{A}$ & $\% 10$ & $22.5 \mathrm{GPa}$ \\
\hline $\mathrm{B}$ & $\% 15$ & $21.25 \mathrm{GPa}$ \\
\hline $\mathrm{C}$ & $\% 25$ & $18.75 \mathrm{GPa}$ \\
\hline
\end{tabular}

Moreover, in order to detect the location of the damage, the dam is divided into ten different zones and they are numbered from one to ten (Figure 5).

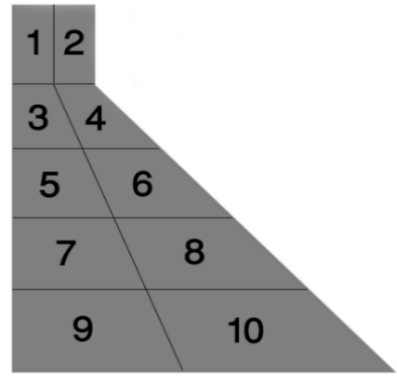

Figure 5. Dividing the Dam into Ten Zones to Detect the Location of the Damage

The location of the measured response of the dam (the history of acceleration) in five equal portions at the height of the dam is represented in Figure 6.

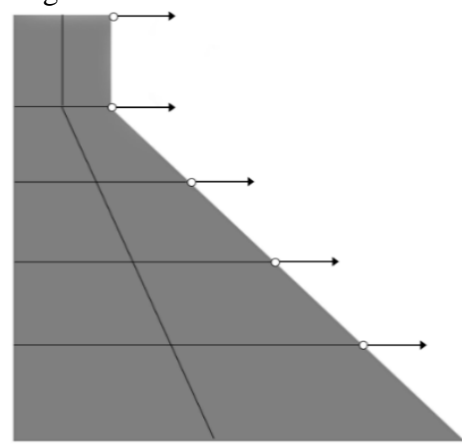

Figure 6. Measuring the Response of the Dam (the Horizontal Component of Acceleration) 
Figure 7 shows an example of the recorded output acceleration for the damaged and undamaged structure. This figure corresponds to $15 \%$ damage index for No. 2 zone on the top of the dam.

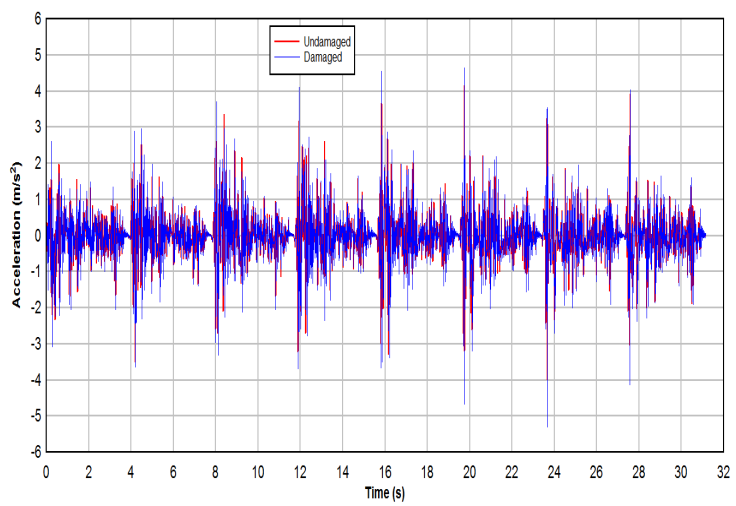

Figure 7. The Acceleration of the Damaged and Undamaged Structure

The following figure (Figure 8) shows the difference between the damaged and undamaged structure responses, which is a result of change in the dynamic response of the dam.

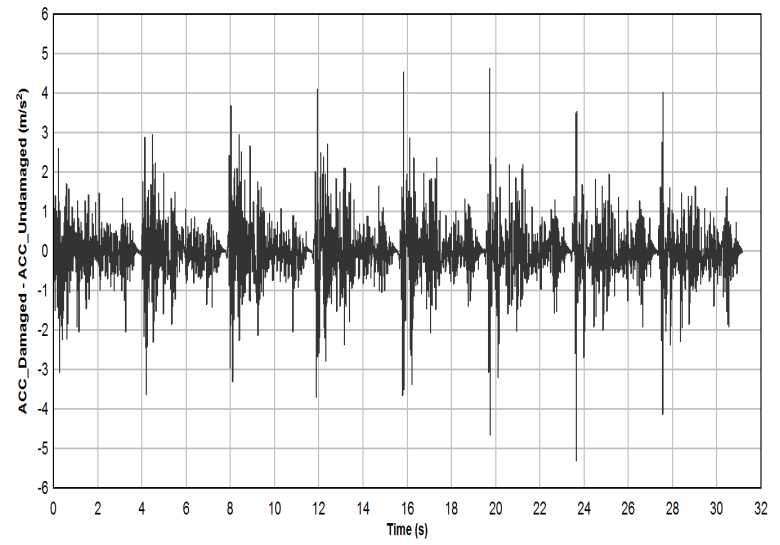

Figure 8. The Effect of Damage as the Difference of Recorded Output Acceleration

After preparing accelerations, by applying Hilbert-Huang Method, the primary natural frequencies of the dam are determined for different modes of damage (different locations and severities of damage). According to Figure 9, it seems that the changes in frequencies can be considered as suitable criteria for the detection of the location and severity of damage in concrete gravity dams.

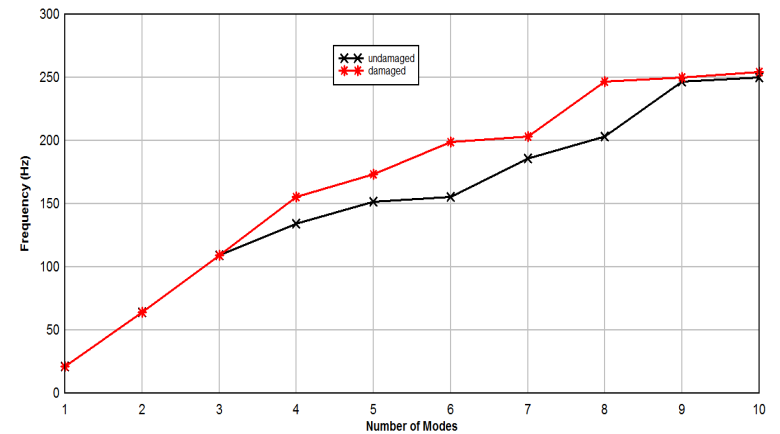

Figure 9. The Difference in Frequency as a Measure of Damage

\subsection{Applying Hilbert-Huang Technique to Output} Accelerations

In this part, the process of applying Hilbert-Huang Method to output signals (history of acceleration) is explained. Figure 10 represents a sample response of the dam that relates to the damage index of $25 \%$ and in points of top, middle and bottom of the structure respectively.

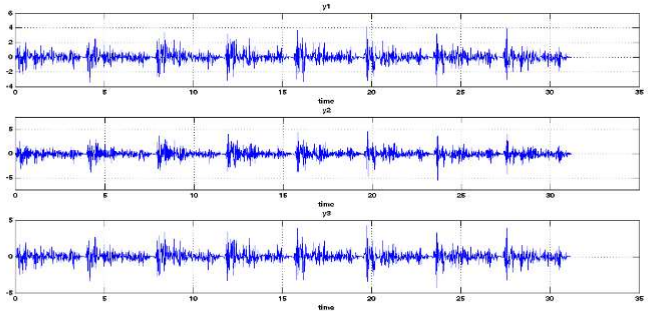

Figure 10. Acceleration of Three Points of Different Heights of the Dam as a Response of the Structure

In the following (Figure 11), empirical decomposition of signals is expressed in three columns. The right hand, the central and the left hand side column relate to the points which are located at the bottom, middle and top of the dam respectively. Furthermore, the first row is related to the original signal and the other rows correspond to the Intrinsic Mode Functions.

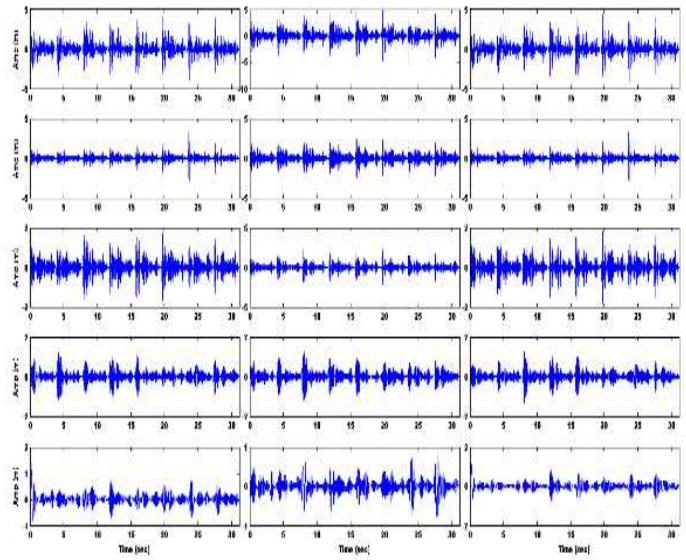

Figure 11. Original Signal and Intrinsic Mode Functions

Figure 12 shows an example of applying Hilbert-Huang Method to the response of the dam. The right hand side represents the largest number of repetition of frequencies in an interval, aside from the first row (which relates to the information about the original signal), the frequency of the second row or the first Intrinsic Mode is $21 \mathrm{~Hz}$ and the frequency of the third row or the second Intrinsic Mode is equal to $64.1 \mathrm{~Hz}$. 


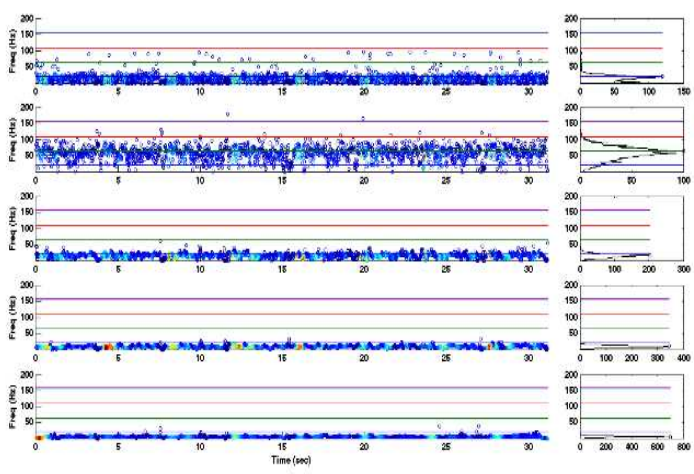

Figure 12. Hilbert Transform on Original Signal and Intrinsic Mode Decomposition of Responses

If the same process is repeated, the changes in the damaged and undamaged frequencies can be as they are shown in Figure 13.

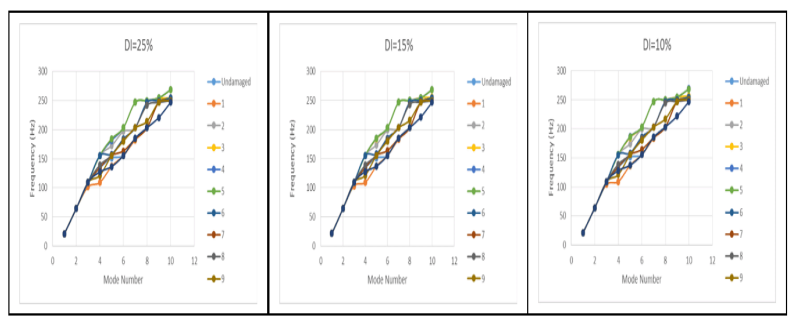

Figure 13. The Frequency Changes of the Damaged and Undamaged Structure

It seems that the three above figures are very similar in appearance. In order to distinguish better these figures, the relative difference (based on severity of $15 \%$ ) between the first two figures is represented in Figure 14.

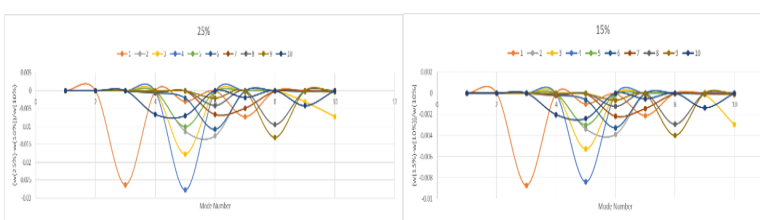

Figure 14. Effect of Damage Severity in Frequency Changes

Considering the previous explanations, it seems that the changes in frequencies can be used as a criterion for damage detection. For this purpose, a parameter, which is called Relative Frequency Error (RFE), is defined to detect the location and severity of the damage in concrete gravity dams as follows:

$\{e\}=\left\{\begin{array}{c}\frac{f_{d}^{1}-f_{u d}^{1}}{f_{u d}^{1}} \\ \frac{f_{d}^{2}-f_{u d}^{2}}{f_{u d}^{2}} \\ \cdot \\ \cdot\end{array}\right\}$
Where, $e$ is the vector of Relative Frequency Error and $f$ is the frequency of system in which the subscripts of $d$ and $u d$ are used for the damaged and undamaged system respectively. Furthermore, the superscript denotes the mode number.

To determine the location of a damaged area according to the Eq. (17), the vector of $\{e\}$ is compared with each vector of the monitored cases $\left\{e^{\prime}\right\}$ and eventually each case, which has a smaller vector size, will determine the location of the damage in the structure.

$\left|\{e\}-\left\{e^{\prime}\right\}\right|_{\min }$

In the following figure, the relative error functions are shown.

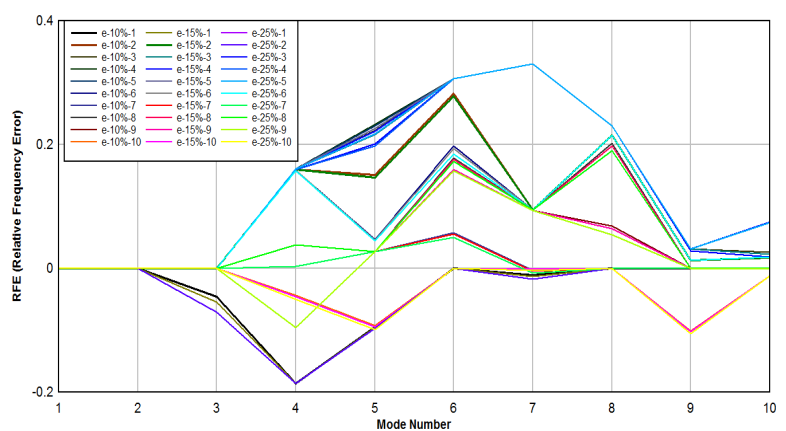

Figure 15. Relative Frequency Error Functions

To determine the severity of damage, based on the smallest value of Relative Frequency Error functions (e $10 \%$, e 15\% and $\mathrm{e}_{25 \%}$ ), two types of damage indexes are proposed as follows:

\subsubsection{Damage Index Proportional to Relative Frequency Error $\left(D I_{1}\right)$ :}

The first step in calculation of this index is determination of two smaller values of $\mathrm{e}_{10 \%}, \mathrm{e}_{15 \%}$ and $\mathrm{e}_{25 \%}$, which leads to the following three cases:

$$
\begin{aligned}
& e_{25 \%}<e_{10 \%} \& \& e_{15 \%}<e_{10 \%} \rightarrow \text { Ca se } 1 \\
& e_{10 \%}<e_{25 \%} \& \& e_{15 \%}<e_{25 \%} \rightarrow \text { Case } 2 \\
& e_{10 \%}<e_{15 \%} \& \& e_{10 \%}<e_{25 \%} \rightarrow \text { Case } 3
\end{aligned}
$$

It is clear that in the first, second and third cases, the damage indices are between $15 \%-25 \%, 10 \%-15 \%$ and $0-10 \%$. If it is assumed that the percentage of damage is between $i \%$ and $j \%$, the following equation can interpolate the damage index between these two percentages:

$$
\begin{aligned}
& \text { If } e_{i}<e_{k} \& \& e_{j}<e_{k} \text { Assume: } e_{k}=\max \left(e_{i}, e_{j}, e_{k}\right) \\
& D I_{1}=\frac{e_{i}}{e_{i}+e_{j}} \times j \%+\frac{e_{j}}{e_{i}+e_{j}} \times i \%
\end{aligned}
$$

To verify the above equation, it should be assumed that the Relative Frequency Error function tends to be zero. In this case, the value of the damage index corresponding to Relative Frequency Error function will be the solution. In other words, if $e_{i}$ in Eq. (19) approaches zero, the damage percentage should be equal to $i \%$. 


$$
\lim _{e_{i} \rightarrow 0}\left(\frac{e_{i}}{e_{i}+e_{j}} \times j \%+\frac{e_{j}}{e_{i}+e_{j}} \times i \%\right)=i \%
$$

\subsubsection{Damage Index Reversely Proportional to Relative Frequency Error $\left(D I_{2}\right)$ :}

It is obvious that as the Relative Frequency Error becomes greater, the corresponding damage index will diverge from the real location of damage. Therefore, the second proposed damage index is defined reversely proportional to the Relative Frequency Error, which can be written as:

$$
D I_{2}=\frac{\sum_{i=1}^{3} \frac{1}{e_{i}} \times i \%}{\sum_{i=1}^{3} \frac{1}{e_{i}}}
$$

In order to control the proposed algorithm, firstly the structure is damaged in various zones with different damage severity; afterwards, by using the proposed method, the damage location and severity will be predicted and compared with the real results. Table 3 represents the samples of this comparison.

Table 3. Comparing the Results of the Predicted and Actual Location and Severity of Damage

\begin{tabular}{|c|c|c|c|c|c|}
\hline & \multicolumn{2}{|c|}{$\begin{array}{c}\text { Location of } \\
\text { Damage }\end{array}$} & \multicolumn{3}{c|}{ Damage Severity } \\
\hline Case & Real & Predicted & Real & $\begin{array}{c}\text { Predicted } \\
\text { with DI }\end{array}$ & $\begin{array}{l}\text { Predicted } \\
\text { with DI }\end{array}$ \\
\hline$a$ & 9 & 9 & $5 \%$ & $6.7 \%$ & $13.3 \%$ \\
\hline$b$ & 7 & 7 & $12 \%$ & $11.91 \%$ & $12.9 \%$ \\
\hline$c$ & 2 & 2 & $18 \%$ & $13.6 \%$ & $13.8 \%$ \\
\hline$d$ & 5 & 5 & $20 \%$ & $21.1 \%$ & $19.1 \%$ \\
\hline
\end{tabular}

\section{CONCLUSION}

Reviewing the past studies shows that the structural damage detection, using signal processing methods, was often applied to simple structural elements such as beams, trusses and frames. However, in this research, Hilbert-Huang Method, as one of the most common techniques in signal processing, is used to predict the location and severity of damage in concrete gravity dams. The results show that the proposed criterion (Relative Frequency Error), which is defined by frequency changes, is properly able to determine the location of damage in the concrete gravity dams (sample results are shown in Table 3). Moreover, to predict the severity of damage in these structures, two damage indexes based on the size of Relative Frequency Error vector are proposed in this study. The results have shown that the first damage index, proportional to Relative Frequency Error which in turn discretely interpolates the percentage of the damage, can predict the actual damage severity of the structure better compared with the second index, inversely proportional to Relative Frequency Error (Table 3). In addition, the new proposed algorithm for damage detection of concrete gravity dams has a simple mathematical equation which is able to make physical sense during the analysis. It should be noted that the presented method in this study may be used for other structures with high degrees of freedom, such as power towers, telecommunication towers, etc., without causing damage to the structure, using the information from the sensors installed on it.

\section{References}

A.Farsayi, R.Abbasnia. 2010. "Wavelet Transform in Damage Detection of Structures." 6th Conference of Iranian civil engineering.

Aldemir, Alper et al. 2015. "Pseudo-Dynamic Testing of a Concrete Gravity Dam."

Alembagheri, Mohammad, and Mohsen Ghaemian. 2013. "Damage Assessment of a Concrete Arch Dam through Nonlinear Incremental Dynamic Analysis." Soil Dynamics and Earthquake Engineering 44: 127-37. http://dx.doi.org/10.1016/j.soildyn.2012.09.010

Allen, J B. 1977. "Short Term Spectral Analysis, Synthesis, and Modification by Discrete Fourier Transform." IEEE Trans. Acoust., Speech, Signal Process. 25(3): 235-38 https://doi.org/10.1109/TASSP.1977.1162950.

Alvandi, A., and C. Cremona. 2006. "Assessment of VibrationBased Damage Identification Techniques." Journal of Sound $\begin{array}{lll}\text { and Vibration } & \text { 292(1-2): } & 179-202\end{array}$ https://doi.org/10.1016/j.jsv.2005.07.036.

Amiri, Gholamreza Ghodrati, and Ehsan Darvishan. 2015. "Damage Detection of Moment Frames Using Ensemble Empirical Mode Decomposition and Clustering Techniques." KSCE Journal of Civil Engineering 19: 1302-11 https://doi.org/10.1007/s12205-015-0415-z.

Balafas, Konstantinos, and Anne S Kiremidjian. 2015. "The Continuous Wavelet Transform as a Stochastic Process for Damage Detection."

Bathe, K. J. 1996. Finite Element Procedures in Engineering Analysis. Prentice Hall.

Bedrosian, E. 1963. "A Product Theorem for Hilbert Transforms." Proceedings of the IEEE 51(5) https://doi.org/10.1109/PROC.1963.2308.

Brincker, Rune, Lingmi Zhang, and Palle Andersen. 2001. "Modal Identification of Output-Only Systems Using Frequency Domain Decomposition." Smart Materials and Structures 10(3): 441-45 https://doi.org/10.1088/0964-1726/10/3/303.

Bukenya, P, P Moyo, H Beushausen, and C Oosthuizen. 2014. "Health Monitoring of Concrete Dams : A Literature Review."

Carolina, North, and Worcester Polytechnic. 1999. "WAVELET-BASED APPROACH FOR STRUCTURAL DAMAGE DETECTION by Z. Hou/ M. Noori/ and R. St. Arnand 3." Engineering.

Chang, Peter C., and S. Chi Liu. 2003. "Recent Research in Nondestructive Evaluation of Civil Infrastructures." Journal of Materials in Civil Engineering 15(3): 298-304. 
Chang, T.Y.P., C.C. Chang, and Y.G. Xu. 1999. "Updating Structural Parameters: An Adaptive Neural Network Approach.” In Structural Health Monitoring 2000, Stanford University, Palo Alto, 379-389 https://doi.org/10.1061/(ASCE)0899-1561(2003)15:3(298).

Chen, Bo, Sheng-lin Zhao, and Peng-yun Li. 2014. "Application of Hilbert-Huang Transform in Structural Health Monitoring: A State-of-the-Art Review." Mathematical Problems in Engineering 2014. http://www.hindawi.com/journals/mpe/2014/317954/abs/ https://doi.org/10.1155/2014/317954.

Chen, Peipei, and Guoxin Zhang. 2014. "Study on the Prevention Measures and Causes of Cracks in Arch Crests of Galleries in High Concrete Dams." 921: 813-19 https://doi.org/10.4028/www.scientific.net/AMR.919-921.813.

Chopra, Anil K. 2014. "EARTHQUAKE ANALYSIS OF CONCRETE DAMS : FACTORS TO BE CONSIDERED.”

Cleary, R Das P W. 2013. "A Mesh-Free Approach for Fracture Modelling of Gravity Dams under Earthquake”, pp. 9-33.

Doebling, S. W., C. R. Farrar, and M. B. Prime. 1998. "A Summary Review of Vibration-Based Damage Identification Methods." The Shock and Vibration Digest 30(2): 91-105 https://doi.org/10.1177/058310249803000201.

Doebling, S W, C R Farrar, M B Prime, and D W Shevitz. 1996. "Damage Identification and Health Monitoring of Structural and Mechanical Systems from Changes in Their Vibration Characteristics: A Literature Review." Other Information: PBD: May 1996 (836): Medium: ED; Size: 132 p.

Fox, C.H.J. 1992. "THE LOCATION OF DEFECTS IN STRUCTURES: ACOMPARISON OF THE USE OF NATURAL FREQUENCY AND MODE SHAPE DATA."

Heylen, W, and S Lammens. 1996. "FRAC: A Consistent Way of Comparing Frequency Response Functions" ed. S Lammens. Proceedings, International Conference on Identification in Engineering, Swansea; 48-57.

Huang, N. E. et al. 2003. "A Confidence Limit for the Empirical Mode Decomposition and Hilbert Spectral Analysis." Proceedings of the Royal Society A: Mathematical, Physical and Engineering Sciences 459(2037): 2317-45 https://doi.org/10.1098/rspa.2003.1123.

Huang, N E. 2005. "Computing Instantaneous Frequency by Normalizing Hilbert Transform." http://www.google.com/patents/US6901353.

Huang, Norden E et al. 1998. "The Empirical Mode Decomposition and the Hilbert Spectrum for Nonlinear and Non-Stationary Time Series Analysis." Proceedings of the Royal Society of London A: Mathematical, Physical and Engineering Sciences 454(1971): 903-95. http://rspa.royalsocietypublishing.org/content/454/1971/903.abs tract.

Humar, J. L, and A. M. Jablonski. 1988. "Boundary Element Reservoir Model for Seismic Analysis of Gravity Dams."
Earthquake Engng. Struct. Dyn. 16(8): 1129-56. http://dx.doi.org/10.1002/eqe.4290160804.

Jeyasehar, C Antony, and K Sumangala. 2006. "Damage Assessment of Prestressed Concrete Beams Using Artificial Neural Network (ANN) Approach." 84: 1709-18 https://doi.org/10.1016/j.compstruc.2006.03.005.

Kyang, K. 2005. Structural Sensivity Analysis and Optimization. Springer.

Lee, J., and S. Kim. 2007. "Structural Damage Detection in the Frequency Domain Using Neural Networks." Journal of Intelligent Material Systems and Structures 18(8): 785-92 https://doi.org/10.1177/1045389X06073640.

Min, Z. H. et al. 2011. "New Methodology for the Application of Vibration-Based Damage Detection Techniques." Structural Control and Health Monitoring 20(May 2011): n/a-n/a.

Pandey, A, M Biswas, and M Samman. 1991. "Damage Detection from Mode Changes in Curvature." Journal of Sound and Vibration 145(2): 321-32 https://doi.org/10.1016/0022460X (91)90595-B.

Patjawit, Anun, and Worsak Kanok-Nukulchai. 2005. "Health Monitoring of Highway Bridges Based on a Global Flexibility Index." Engineering Structures 27(9): 1385-91 https://doi.org/10.1016/j.engstruct.2005.04.003.

R. A. Osegueda, P. D. DSouza, and Qiang Y. J. 1992. "Damage Evaluation of Offshore Structures Using Frequency Shifts." ASMEPVP Conference: $31-37$.

Ramezani \& Bahar. 2010. "Mode Shape Identification Using Modified Hilbert-Huang Transform." 5th Conference of Iranian civil engineering.

Richard, E. 2010. Processing, Fast Algorithms for Aignal. Cambridge https://doi.org/10.1002/9780470976401.ch4.

Rilling, Gabriel, Patrick Flandrin, Paulo Gon, and De Lyon. 2003. "On Empirical Mode Decomposition and Its Algorithms." IEEEEURASIP Workshop on Nonlinear Signal and Image Processing NSIP 3: 8-11. http://perso.enslyon.fr/patrick.flandrin/NSIP03.pdf.

Ruotolo, R., and C. Surace. 1997. "Damage Assessment of Multiple Cracked Beams: Numerical Results and Experimental Validation." Journal of Sound and Vibration 206: 567-88 https://doi.org/10.1006/jsvi.1997.1109.

Rusch, T, R Sankar, and J Scharf. 1995. "Signal Processing Methods for Pulse Oximetry." Compt. Biol. Med 26(2): 143-59 https://doi.org/10.1016/0010-4825 (95)00049-6.

Rytter, Anders. 1993. "Vibrational Based Inspection of Civil Engineering Structures." Dept. of Building Technology and Structural Engineering, Aalborg University.

Salawu, O.S. 1997. "Detection of Structural Damage through Changes in Frequency: A Review." Engineering Structures 19(9): 718-23 https://doi.org/10.1016/S0141-0296 (96)001496. 
Scharf, L1. 1991. "Statistical Signal Processing." http://www.ulb.tu-darmstadt.de/tocs/21055823.pdf.

Sethi, Rabinarayan, S.K. Senapati, and Dayal R. Parhi. 2014. "Structural Damage Detection by Fuzzy Logic Technique." Applied Mechanics and Materials 592-594: 1175-79 https://doi.org/10.4028/www.scientific.net/AMM.592-

594.1175 .

Stubbs, Norris, and Jeong Kim. 1992. "An Efficient and Robust Algorithm for Damge Localization in Offshore Platform." International Journal of Offshore and Polar Engineering 5(1053-5381).

Tang, J.-P. et al. 2011. "A Case Study of Damage Detection in Benchmark Buildings Using a Hilbert-Huang Transform-Based Method." Journal of Vibration and Control 17(4): 623-36 https://doi.org/10.1177/1077546309360053.

Zhu, Futao, and Yunjie Wu. 2014. "A Rapid Structural Damage Detection Method Using Integrated ANFIS and Interval Modelling Technique." Applied Soft Computing Journal 25: 473-84 https://doi.org/10.1016/j.asoc.2014.08.043. 